\title{
A STATE SPACE ERROR ESTIMATE FOR POD-DEIM NONLINEAR MODEL REDUCTION *
}

\author{
SAIFON CHATURANTABUT AND DANNY C. SORENSEN †
}

\begin{abstract}
This paper derives state space error bounds for the solutions of reduced systems constructed using Proper Orthogonal Decomposition (POD) together with the Discrete Empirical Interpolation Method (DEIM) recently developed in [4] for nonlinear dynamical systems. The resulting error estimates are shown to be proportional to the sums of the singular values corresponding to neglected POD basis vectors both in Galerkin projection of the reduced system and in the DEIM approximation of the nonlinear term. The analysis is particularly relevant to ODE systems arising from spatial discretizations of parabolic PDEs. The derivation clearly identifies where the parabolicity is crucial. It also explains how the DEIM approximation error involving the nonlinear term comes into play.
\end{abstract}

Key words. Nonlinear Model Reduction, Proper Orthogonal Decomposition, Empirical Interpolation Methods, Nonlinear Partial Differential Equations

AMS subject classifications. 65L02, 65M02

1. Introduction. Model order reduction (MOR) can effectively reduce computational costs for simulation of dynamical systems. Such reductions are usually achieved through some approximation scheme that produces a reduced dimension dynamical system with nearly the same response characteristics as the original. Although there are many instances where the primary interest is in preserving inputoutput relations, there is generally an interest in obtaining good approximations to the state variables as well. Here, we analyze the state approximation error for the Discrete Empirical Interpolation Method (DEIM) which is a dimension reduction method for large systems of nonlinear ordinary differential equations (ODEs). In particular, we analyze the DEIM state approximation error of systems of ODEs arising from a spatial discretization of time dependent parabolic partial differential equations (PDEs) (e.g., convection-diffusion-reaction equations). We provide a bound on the global state space approximation error as well as a uniform bound on the pointwise error over a specified time interval. The analysis shows precisely where the parabolicity is crucial and it shows exactly how the DEIM approximation error involving the nonlinear term comes into play. The ultimate result lends rigor to the heuristic notion that the error in trajectory approximation should be proportional to the first neglected singular value in the truncated singular value decomposition (SVD) used to obtain the reduced basis.

The DEIM can be viewed as a modification of proper orthogonal decomposition (POD), which constructs a reduced basis from a truncated SVD of a matrix of sampled trajectory vectors (snapshots). The standard POD-Galerkin approach can provide a significant reduction in the number of variables, but for many problems (including the broad class of ODEs coming from spatial discretization of convection-diffusionreaction equations), the complexity of evaluating the reduced dimension nonlinear terms remains that of the original high dimensional system. As a result, the reduced system is just as expensive to solve as the original, and there is little or no reduction in

\footnotetext{
*Work supported in part by NSF grant CCF-1017401 and by AFOSR grant FA9550-09-1-0225.

†DEPARTMENT OF COMPUTATIONAL AND APPLIED MATHEMATICS, MS-134, RICE UNIVERSITY, 6100 MAIN STREET, HOUSTON, TEXAS 77005-1892, USA. SC3@RICE.EDU, SORENSEN@RICE.EDU
} 
simulation time. The DEIM resolves this complexity issue. It does so by replacing the orthogonal projection of POD with an interpolatory projection. The result enables the nonlinear term to be evaluated at just a few selected component functions and thus greatly reduces the complexity.

In $[16,17]$, Kunish and Volkwein derive error estimates for a POD reduced system in a function space setting for a class of nonlinear parabolic PDEs. They analyzed solution snapshots as well as the POD basis in general Hilbert space. Kunish and Volkwein also considered a snapshot set that included finite difference quotients of the snapshots. The approximation errors were expressed as the contributions from the POD subspace approximation error and from time discretization error. The theoretical results in [17] provide asymptotic error estimates that do not depend on the snapshot set and demonstrate the effect of two different time discretizations used to produce the set of snapshots and for the numerical integration of the reduced system. Nonlinear problems with a Lipschitz continuous nonlinearity are considered in [16]. and extended to the Navier-Stokes equations in [17]. Our analysis of the DEIM follows the Kunish-Volkwein approach in [30] to obtain error bounds for POD reduced system of ODEs.

Several authors have worked specifically in the ODE setting with snapshots and solutions residing in finite dimensional Euclidean space $[29,8,19,24,13,14]$. In [19], the authors applied the dual-weighted-residual method which uses the solution of a dual or adjoint system to obtain an error estimate for the solutions from POD reduced models of nonlinear systems. In [24], the error bounds of solutions from a POD reduced system were derived and the effects of small perturbations on the set of snapshots used for constructing the POD basis were studied. Subsequent work [14] proposed an alternative error estimation based on an adjoint method combined with the method of small sample statistical condition estimation. It also analyzed further the effect of perturbations in both the initial conditions and parameters on the resulting POD reduced system. However, the analysis in [14] is based on linearization, and hence, large perturbations may require some knowledge of the solution of the perturbed system. Additional error estimates for reduced systems constructed from projection methods, particularly those based on POD-Galerkin technique, can also be found in $[29,8,19,13]$ as reviewed in [14].

DEIM is a discrete variant of the Empirical Interpolation Method (EIM) proposed by Barrault, Maday, Nguyen and Patera in [1] where an a priori error bound and an a posteriori error estimate of EIM coefficient-function approximation with a basis from the proposed greedy selection process were given. A more general a posteriori error bound (but more expensive to compute) was also recently proposed in [7]. The incorporation of these error estimates [1] for EIM function approximation into the reduced basis framework [23, 25, 22] to obtain error bounds of the outputs from nonlinear systems or linear systems with nonaffine parameter dependence can be found in $[21,20,10]$.

Here we extend the error analysis of Kunish and Volkwein for POD reduced systems to the POD-DEIM reduced systems presented in [4] for ODEs with Lipschitz continuous nonlinearities. As mentioned previously, DEIM improves the efficiency of the POD approximation and achieves a complexity reduction of the nonlinear term with a complexity proportional to the number of reduced variables. Evaluating the approximate nonlinear term does not require a prolongation of the reduced state variables back to the original high dimensional space as required in the POD approximation and it avoids evaluation of the original high dimensional nonlinearity. 
Recently, DEIM has been successfully used for nonlinear model reduction in the application of neural modeling of full Hodgkin-Huxley models for realistic spiking neurons [15] and in the application of two-phase miscible flow in porous media with varying Peclet number, both with and without chemistry at the interface of the different fluids [3]. Galbally et al. [9] applied a closely related method called masked projection and obtained reductions of order $10^{5}$ in both dimension and CPU time for a convection diffusion reaction problem. An error bound for the DEIM approximation of a nonlinear function is given in [4] which shows it is nearly as accurate as the optimal orthogonal projection in POD approximation. This error bound is used in the analysis presented here to establish the global accuracy of the POD-DEIM reduced system. Hinze and Kunkel [12] apply the DEIM to a POD model order reduction of drift-diffusion equations in electrical networks. Their results also verify this preservation of accuracy and they achieve significant reduction in simulation times over the original POD reduced system.

To isolate the effect of time discretization error, we first compare the exact solutions of the resulting POD-DEIM reduced system with the exact solutions of the original full-order system. Of course, the exact solutions are generally impossible to obtain in practice. We therefore also compare the numerical solutions from the discretized reduced system obtained from the same numerical scheme as the original discretized system in which the snapshots are collected. Our analysis is illustrated here for the case when the implicit Euler scheme is used for time discretization. Similar error bounds can be obtained for other discretization schemes. We shall use $\|\cdot\|$ to denote the 2-norm in Euclidean space throughout this paper. The 2-norm error estimates presented here are shown to be proportional to the sums of the singular values corresponding to neglected POD basis vectors both in Galerkin projection of the reduced system and in DEIM approximation of the nonlinear term. The separate POD basis used in DEIM to approximate the nonlinearity is very closely related KunishVolkwein's inclusion of finite difference snapshots $\left(\mathbf{y}_{j+1}-\mathbf{y}_{j}\right) / h$ into the snapshot set, since $\left(\mathbf{y}_{j+1}-\mathbf{y}_{j}\right) / h \approx \dot{\mathbf{y}}\left(t_{j}\right)=\mathbf{f}\left(\mathbf{y}_{j}\right)$, where $\mathbf{y}_{j} \approx \mathbf{y}\left(t_{j}\right)$ and $\dot{\mathbf{y}}=\mathbf{f}(\mathbf{y})$.

2. Problem formulation. We shall first consider systems of nonlinear ODEs of the form:

$$
\frac{d}{d t} \mathbf{y}(t)=\mathbf{A y}(t)+\mathbf{F}(t, \mathbf{y}(t)), \quad \mathbf{y}(0)=\mathbf{y}_{0}, \quad \text { for } t \in[0, T]
$$

where the matrix $\mathbf{A}$ is constant and the nonlinear function $\mathbf{F}$ is assumed to be uniformly Lipschitz continuous with respect to the second argument with Lipschitz constant $L_{f}>0$, i.e.,

$$
\left\|\mathbf{F}\left(t, \mathbf{y}_{1}\right)-\mathbf{F}\left(t, \mathbf{y}_{2}\right)\right\| \leq L_{f}\left\|\mathbf{y}_{1}-\mathbf{y}_{2}\right\|,
$$

for all $t \in[0, T]$. In the POD-DEIM approach [4], two POD bases are derived. The first of these consists of the columns of the $n \times k$ orthogonal matrix $\mathbf{V}$ obtained from a truncated SVD of the trajectory snapshot matrix $\mathbb{Y}=\left[\mathbf{y}_{1}, \mathbf{y}_{2}, \ldots, \mathbf{y}_{n_{s}}\right]$ while the second one consists of the columns of the $n \times m$ orthogonal matrix $\mathbf{U}$ obtained from a truncated SVD of the nonlinear snapshot matrix $\mathbb{F}=\left[\mathbf{f}_{1}, \mathbf{f}_{2}, \ldots, \mathbf{f}_{n_{s}}\right]$ where $\mathbf{y}_{j} \approx \mathbf{y}\left(t_{j}\right)$ and $\mathbf{f}_{j}=\mathbf{F}\left(t_{j}, \mathbf{y}_{j}\right)$. The corresponding POD-DEIM reduced system is constructed by applying Galerkin projection on the space spanned by columns of POD basis matrix $\mathbf{V} \in \mathbb{R}^{n \times k}, k \ll n$, and then applying DEIM approximation to the nonlinear function using interpolation projection onto the column space of the POD 
basis matrix $\mathbf{U} \in \mathbb{R}^{n \times m}, m \ll n$. The resulting reduced system is then given by

$$
\frac{d}{d t} \widehat{\mathbf{y}}(t)=\widehat{\mathbf{A}} \widehat{\mathbf{y}}(t)+\mathbf{V}^{T} \mathbb{P} \mathbf{F}(t, \mathbf{V} \widehat{\mathbf{y}}(t)), \quad \widehat{\mathbf{y}}(0)=\mathbf{V}^{T} \mathbf{y}_{0}, \quad \text { for } t \in[0, T]
$$

where $\widehat{\mathbf{A}}:=\mathbf{V}^{T} \mathbf{A} \mathbf{V}, \mathbb{P}:=\mathbf{U}\left(\mathbf{P}^{T} \mathbf{U}\right)^{-1} \mathbf{P}^{T}$, and $\mathbf{P} \in \mathbb{R}^{n \times m}$ is a matrix whose columns come from some selected columns of the identity matrix corresponding to the DEIM indices. Notice that if $m=n$, then $\mathbb{P}$ is is equal to the $n$-by- $n$ identity matrix and the system in (2.3) is just a reduced system constructed by the standard POD-Galerkin approach.

Recall that the Lipschitz continuity assumption on $\mathbf{F}:[0, T] \times D \rightarrow \mathbb{R}^{n}, D \subseteq \mathbb{R}^{n}$ in the original system (2.1) will guarantee the existence and uniqueness of the solution from the original system (by, e.g., Picard-Lindelöf theorem). The Lipschitz continuity of $\mathbf{F}$ is inherited by the reduced order nonlinear term $\widehat{\mathbf{F}}(t, \widehat{\mathbf{y}}(t)):=\mathbf{V}^{T} \mathbb{P} \mathbf{F}(t, \mathbf{V} \widehat{\mathbf{y}}(t))$, since $\left\|\widehat{\mathbf{F}}\left(t, \widehat{\mathbf{y}}_{1}(t)\right)-\widehat{\mathbf{F}}\left(t, \widehat{\mathbf{y}}_{2}(t)\right)\right\|=\left\|\mathbf{V}^{T} \mathbb{P} \mathbf{F}\left(t, \mathbf{V} \widehat{\mathbf{y}}_{1}(t)\right)-\mathbf{V}^{T} \mathbb{P} \mathbf{F}\left(t, \mathbf{V} \widehat{\mathbf{y}}_{2}(t)\right)\right\| \leq$ $L_{f}\|\mathbb{P}\|\left\|\widehat{\mathbf{y}}_{1}(t)-\widehat{\mathbf{y}}_{2}(t)\right\|$, for all $t \in[0, T]$, where $\|\mathbb{P}\|$ is a bounded constant as shown in [4] and we have used the fact that $\mathbf{V}$ has orthonormal columns. Thus, existence and uniqueness of the solution to the POD-DEIM reduced system (2.3) will also be inherited.

The solution $\mathbf{y}(t)$ of the original full-order system (2.1) can be approximated by $\mathbf{V} \widehat{\mathbf{y}}$ where $\widehat{\mathbf{y}}$ is the solution from the POD-DEIM reduced system (2.3). The accuracy of this approximation therefore can be measured by considering the error $\|\mathbf{y}(t)-\mathbf{V} \widehat{\mathbf{y}}(t)\|$ for $t \in[0, T]$. The bounds for this DEIM state space error will be the main focus of our analysis. However, the derivation of the error bounds which will be presented later in this paper can be applied to the case when other matrices with orthonormal columns are used in place of these POD basis matrices. This derivation also can be extended to a more general class of parametrized ODE systems. We shall begin with a brief review, along with the development of some relevant notation, for POD and DEIM approximation.

2.1. Proper Orthogonal Decomposition (POD). POD constructs an orthonormal basis that can represent dominant characteristics of the space of expected solutions which is defined as Range( $\mathbb{Y})$, the span of the snapshots. Recall that snapshots are numerically sampled values of the trajectory $\mathbf{y}(\cdot)$ at particular time steps and at particular parameter values. Assuming $r=\operatorname{rank}\{\mathbb{Y}\}$. POD gives an optimal set of basis vectors $\left\{\mathbf{v}_{i}\right\}_{i=1}^{k} \subset \mathbb{R}^{n}$ minimizing the mean square error associated with approximating these snapshots for $k \leq r$. In particular, the POD basis matrix $\mathbf{V}=\left[\mathbf{v}_{1}, \ldots, \mathbf{v}_{k}\right] \in \mathbb{R}^{n \times k}$ solves the minimization problem:

$$
\min _{\operatorname{rank}\{\mathbf{V}\}=k} \sum_{j=1}^{n_{s}}\left\|\mathbf{y}_{j}-\mathbf{V} \mathbf{V}^{T} \mathbf{y}_{j}\right\|^{2}, \quad \text { s.t. } \quad \mathbf{V}^{T} \mathbf{V}=\mathbf{I}_{k} .
$$

We recommend [17] for more detail on POD. Here, we shall work in a finite dimensional setting where POD is essentially the same as a truncated singular value decomposition (SVD). Specifically, a POD basis of dimension $k$ for (2.4) is just a set of left singular vectors corresponding to the first $k$ dominant singular values of $\mathbb{Y}$, the snapshot matrix. The minimum 2-norm error from approximating the snapshots using the POD basis is given by

$$
\sum_{j=1}^{n_{s}}\left\|\mathbf{y}_{j}-\mathbf{V} \mathbf{V}^{T} \mathbf{y}_{j}\right\|^{2}=\sum_{i=k+1}^{r} \sigma_{i}^{2}=\sum_{i=k+1}^{r} \lambda_{i},
$$


for $k<r$, where $\mathbf{V}=\left[\mathbf{v}_{1}, \ldots, \mathbf{v}_{k}\right] \in \mathbb{R}^{n \times k} ; \mathbf{v}_{1}, \mathbf{v}_{1}, \ldots, \mathbf{v}_{r} \in \mathbb{R}^{n}$ are the singular vectors corresponding to the nonzero singular values $\sigma_{1} \geq \sigma_{2} \geq \ldots \geq \sigma_{r}>0$ of $\mathbb{Y}$; and $\lambda_{i}=\sigma_{i}^{2}, i=1, \ldots, r$. In the large scale setting, we recommend using the MATLAB routine svds (or ARPACK) to just compute the dominant singular values and vectors of $\mathbb{Y}$. If $n \leq n_{s}$ one only need compute matrix-vector products of the form $\mathbf{w}=\mathbb{Y}\left(\mathbb{Y}^{T} \mathbf{v}\right)$, while if $n>n_{s}$, it is usually more efficient to compute the dominant singular values and vectors of $\mathbb{Y}^{T}$ which will only require matrix-vector products of the form $\mathbf{w}=\mathbb{Y}^{T}(\mathbb{Y} \mathbf{v})$.

Ultimately, we shall analyze the discrete setting and include effects of numerical approximation. However, it is illuminating to first consider an ideal setting where we assume the entire continuous trajectory is available on the interval $[0, T]$. This will isolate the contribution to the error resulting solely from application of the PODDEIM model reduction technique without introducing the effect from discretization error or the choice of snapshots. Our analysis will require the POD basis to satisfy the following the minimization problem:

$$
\min _{\operatorname{rank}\{\mathbf{V}\}=k} \int_{0}^{T}\left\|\mathbf{y}(t)-\mathbf{V} \mathbf{V}^{T} \mathbf{y}(t)\right\|^{2} d t, \quad \text { s.t. } \quad \mathbf{V}^{T} \mathbf{V}=\mathbf{I}_{k},
$$

as in, e.g. $[16,17,30]$. It is well known [17] that the POD basis which solves (2.6) is the set of first $k$ dominant eigenvectors of the symmetric matrix $\mathbf{R}:=$ $\int_{0}^{T} \mathbf{y}(t) \mathbf{y}(t)^{T} d t \in \mathbb{R}^{n \times n}$. Using the notation established in [17], let $r=\operatorname{rank}\{\mathbf{R}\}$ and let $\lambda_{1}^{\infty} \geq \lambda_{2}^{\infty} \geq \ldots, \geq \lambda_{r}^{\infty}>0$ be the nonzero eigenvalues of $\mathbf{R}$ with the corresponding eigenvectors $\mathbf{v}_{1}, \mathbf{v}_{2}, \ldots, \mathbf{v}_{r} \in \mathbb{R}^{n}$. Then, as in (2.5), POD basis matrix for (2.6) is $\mathbf{V}=\left[\mathbf{v}_{1}, \ldots, \mathbf{v}_{k}\right] \in \mathbb{R}^{n \times k}$ for $k<r$, and the corresponding minimum 2-norm error is

$$
\int_{0}^{T}\left\|\mathbf{y}(t)-\mathbf{V V}^{T} \mathbf{y}(t)\right\|^{2} d t=\sum_{i=k+1}^{r} \lambda_{i}^{\infty} .
$$

The connection between (2.5) and (2.7) was demonstrated in [17] when the sampled snapshots used for (2.4) are sufficiently dense in $[0, T]$. In particular, for fixed time step, $\sum_{i=k+1}^{r} \lambda_{i} \leq 2 \sum_{i=k+1}^{r} \lambda_{i}^{\infty}$ when $n_{s}>\bar{n}_{s}$ for some sufficient large value $\bar{n}_{s}$.

The application of DEIM also requires use of the POD basis matrix $\mathbf{U} \in \mathbb{R}^{n \times m}$ of nonlinear snapshots $\mathbf{f}(t):=\mathbf{F}(t, \mathbf{y}(t)), t \in[0, T]$. For the nonlinear snapshot matrix $\mathbb{F}=\left[\mathbf{f}_{1}, \ldots, \mathbf{f}_{n_{s}}\right] \in \mathbb{R}^{n \times n_{s}}, \mathbf{f}_{j}=\mathbf{f}\left(t_{j}\right), t_{j} \in[0, T]$, the columns of $\mathbf{U}$ are the eigenvectors of $\mathbb{F F}^{T}$ corresponding to the first $m$ dominant eigenvalues $s_{1} \geq \ldots \geq s_{m} \geq \ldots \geq s_{r_{s}}>$ $0, r_{s}:=\operatorname{rank}\{\mathbb{F}\}$. The 2 -norm error is given by

$$
\sum_{j=1}^{n_{s}}\left\|\mathbf{f}_{j}-\mathbf{U} \mathbf{U}^{T} \mathbf{f}_{j}\right\|^{2}=\sum_{i=m+1}^{r_{s}} s_{i}
$$

As with the trajectory, we also consider the POD basis matrix $\mathbf{U}$ satisfying the condition (2.6) for the entire set of continuous nonlinear snapshots $\mathbf{f}(t), t \in[0, T]$. Let $s_{1}^{\infty} \geq s_{2}^{\infty} \geq \ldots \geq s_{r_{s}}^{\infty}>0$ be the $r_{s}$ nonzero eigenvalues of $\int_{0}^{T} \mathbf{f}(t) \mathbf{f}(t)^{T} d t \in \mathbb{R}^{n \times n}$ so that we have

$$
\int_{0}^{T}\left\|\mathbf{f}(t)-\mathbf{U} \mathbf{U}^{T} \mathbf{f}(t)\right\|^{2} d t=\sum_{i=m+1}^{r_{s}} s_{i}^{\infty}
$$


2.2. The Discrete Empirical Interpolation Method (DEIM). DEIM was introduced in [4] to provide an approximation to the nonlinear terms of ODE systems in a form that enables precomputation of certain matrices so that the computational cost of evaluating the nonlinear terms is greatly decreased and is also independent of original dimension $n$. DEIM is a discrete variant of the Empirical Interpolation Method (EIM) proposed by Barrault, Maday, Nguyen and Patera in [1]. Evaluating the approximate nonlinear term from DEIM does not require a prolongation of the reduced state variables back to the original high dimensional state space as is required to evaluate the nonlinearity in the original POD approximation. Only a few entries of the original nonlinear term corresponding to the specially selected interpolation indices from the DEIM must be evaluated at each time step.

Let $\mathbf{f}: \mathscr{D} \mapsto \mathbb{R}^{n}$ be a nonlinear vector-valued function with $\mathscr{D} \subset \mathbb{R}^{d}$, for some positive integer $d$. Let $\mathbf{U}=\left[\mathbf{u}_{1}, \ldots, \mathbf{u}_{m}\right] \in \mathbb{R}^{n \times m}$ with $\operatorname{rank}\{\mathbf{U}\}=m$. The DEIM approximation of order $m \leq n$ in $\operatorname{span}\{\mathbf{U}\}$ for $\mathbf{f}$ is given by

$$
\widehat{\mathbf{f}}(\tau):=\mathbb{P} \mathbf{f}(\tau),
$$

where $\mathbb{P}=\mathbf{U}\left(\mathbf{P}^{T} \mathbf{U}\right)^{-1} \mathbf{P}^{T}$ and $\mathbf{P}=\left[\mathbf{e}_{\wp_{1}}, \ldots, \mathbf{e}_{\wp_{m}}\right] \in \mathbb{R}^{n \times m}$ with $\left\{\wp_{1}, \ldots, \wp_{m}\right\}$ being the output index set from Algorithm 1 with the input basis $\left\{\mathbf{u}_{i}\right\}_{i=1}^{m}$; and the vector $\mathbf{e}_{\wp_{j}}=[0, \ldots, 0,1,0, \ldots, 0]^{T} \in \mathbb{R}^{n}$ denoting the $\wp_{j}$-th column of the $n$-by- $n$ identity matrix, i.e., having all zero entries except one at the entry $\wp_{j}$, for $j=1, \ldots, m$. Note that, for general definition of the DEIM approximation (2.10), the matrix $\mathbf{U}$ is not required to have orthonormal columns as in the previous sections. The notation max

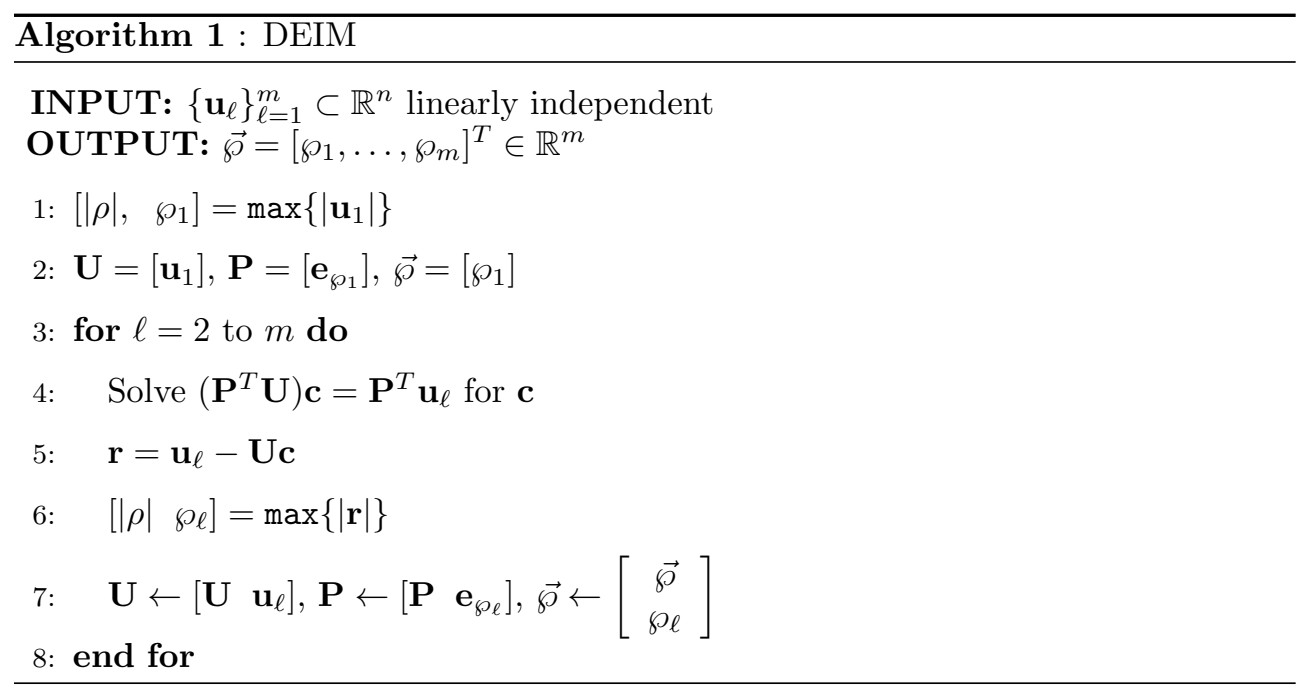

in Algorithm 1 is the same as the function max in MAtrab. Thus, $\left[|\rho|, \wp_{\ell}\right]=\max \{|\mathbf{r}|\}$ implies $|\rho|=\left|r_{\wp_{\ell}}\right|=\max _{i=1, \ldots, n}\left\{\left|r_{i}\right|\right\}$, with the smallest index taken in case of a tie. The interpolation indices selection process of the DEIM may be interpreted as locally limiting the growth of the matrix $\left(\mathbf{P}^{T} \mathbf{U}\right)^{-1}$. When $\mathbf{U}$ has orthonormal columns, there is a bound on the approximation error of the interplant as given below (see Lemma 3.2 in [4]).

LEMma 2.1. Using the notations defined earlier, for $\mathbf{U}^{T} \mathbf{U}=\mathbf{I}$, the bound for the approximation error $\|\mathbf{f}-\widehat{\mathbf{f}}\|$ is given by

$$
\mathbf{f}(\tau)-\widehat{\mathbf{f}}(\tau)=\mathbb{P} \mathbf{w}(\tau) \quad \text { and } \quad\|\mathbf{f}(\tau)-\widehat{\mathbf{f}}(\tau)\| \leq \mathbf{C}_{m} \mathscr{E}_{*}(\mathbf{f})
$$


where $\mathbf{w}(\tau):=\left(\mathbf{I}-\mathbf{U U}^{T}\right) \mathbf{f}(\tau)$,

$$
\mathbf{C}_{m}:=\left\|\left(\mathbf{P}^{T} \mathbf{U}\right)^{-1}\right\| \text { and } \quad \mathscr{E}_{*}(\mathbf{f})=\left\|\left(\mathbf{I}-\mathbf{U U}^{T}\right) \mathbf{f}\right\| .
$$

$\mathscr{E}_{*}(\mathbf{f})$ is the error of the best 2-norm approximation for $\mathbf{f}$ from the space Range( $\left.\mathbf{U}\right)$ and the constant $\mathbf{C}_{m}$ is bounded by

$$
\mathbf{C}_{m} \leq(1+\sqrt{2 n})^{m-1}\left\|\mathbf{u}_{1}\right\|_{\infty}^{-1} .
$$

The invertibility of $\mathbf{P}^{T} \mathbf{U}$ at each iteration of DEIM procedure follows from the boundedness of its inverse as shown (2.13) which was originally derived in [4]. The a priori bound (2.13) is, of course, useless in practice as it is a gross overestimate. In our computations, we simply evaluate $\left\|\left(\mathbf{P}^{T} \mathbf{U}\right)^{-1}\right\|_{2}$ and use this as an a posteriori estimate. This quantity has been on the order of 100 or less in all of the experiments we have conducted. The error bound (2.11) will be used in the next section to analyze the accuracy of the state variables in the POD-DEIM reduced system.

3. Error analysis of POD-DEIM reduced system. This section develops a bound on the state approximation error for numerical solutions obtained from the POD-DEIM reduced system. The derivation will involve an application of the logarithmic norm [5] and the integral form of Gronwall's lemma [11, 2]. We will consider the logarithmic norm of $\mathbf{A} \in \mathbb{C}^{n \times n}$ with respect to the 2-norm defined as

$$
\mu(A):=\lim _{h \rightarrow 0^{+}} \frac{\|\mathbf{I}+h \mathbf{A}\|_{2}-1}{h},
$$

which has an explicit expression suitable for the calculation given by

$$
\mu(A)=\max \left\{\mu: \mu \in \sigma\left(\left[\mathbf{A}+\mathbf{A}^{*}\right] / 2\right)\right\},
$$

where $\sigma\left(\left[\mathbf{A}+\mathbf{A}^{*}\right] / 2\right)$ is the set of eigenvalues of the Hermitian part $\left[\mathbf{A}+\mathbf{A}^{*}\right] / 2$ of A. A well-known property of logarithmic norm that will be used here is

$$
\left\|e^{\mathbf{A} t}\right\| \leq e^{\mu(\mathbf{A}) t} .
$$

for $t \geq 0$ (see, e.g. $[5,28,18]$ ). It is straightforward to show that

$$
\mu(\widehat{\mathbf{A}}) \leq \mu(\mathbf{A}),
$$

where $\widehat{\mathbf{A}}=\mathbf{V}^{T} \mathbf{A V} \in \mathbb{R}^{k \times k}$ and $\mathbf{V} \in \mathbb{R}^{n \times k}, \mathbf{V}^{T} \mathbf{V}=\mathbf{I}$. Hence, (3.3) and (3.4) give

$$
\left\|e^{\widehat{\mathbf{A}} t}\right\| \leq e^{\mu(\mathbf{A}) t} .
$$

The logarithmic norm was introduced by Dahlquist [5] to provide a mechanism for bounding the growth of solution to a linear dynamical system of the form

$$
\dot{\mathbf{y}}(t)=\mathbf{A y}(t)+\mathbf{r}(t)
$$

whenever $\mathbf{r}$ is a bounded function of $t$. For $t \geq 0$ the norm of $\mathbf{y}$ satisfies the differential inequality

$$
\frac{d}{d t}\|\mathbf{y}(t)\| \leq \mu(\mathbf{A})\|\mathbf{y}(t)\|+\|\mathbf{r}(t)\|
$$


As explained by Söderlind [27], the bound (3.6) is able to distinguish between forward and reverse time and it may also be able to distinguish between stable and unstable systems. In fact, $\mu(\mathbf{A})$ may be negative and when it is, the system is certain to be stable. The opposite assertion ( $\mathbf{A}$ stable implies $\mu(\mathbf{A})<0$ ) is not true. The nonnormal matrix $\left[\begin{array}{ll}\lambda & 1 \\ 0 & \lambda\end{array}\right]$ provides a counterexample when $-.5<\operatorname{Real}(\lambda)<0$. More details on logarithmic norms can be found in e.g. [5, 28, 6, 27]. Next, we shall derive bounds on the state approximation error provided by POD-DEIM solutions in two different settings: one involves the ideal case involving the full trajectory of the ODE system while the other one applies to the reduced system derived from snapshots obtained via numerical solution of the ODE system.

3.1. Error bounds in ODE setting. This section compares the solution $\mathbf{y}(t)$ from the original full-order system to the approximation $\mathbf{V} \widehat{\mathbf{y}}(t)$ where $\widehat{\mathbf{y}}$ is the solution of the POD-DEIM reduced system (2.3). We shall show that the resulting error bounds in 2-norm can be approximated by the sums of the singular values corresponding to neglected POD basis vectors both in Galerkin projection of the reduced system and in DEIM approximation of the nonlinear term.

THEOREM 3.1. Let $\mathbf{y}(t)$ be the solution of the original full-order system (2.1) and $\widehat{\mathbf{y}}$ be the solution of the POD-DEIM reduced system (2.3), for $t \in[0, T]$. Let $\mu=\mu(\mathbf{A})$ be the logarithmic norm defined in (3.1) and assume that $\mathbf{F}(t, \mathbf{y})$ in (2.1) is Lipschitz continuous in the second argument with Lipschitz constant $L_{f}$ as in (2.2). Then

$$
\int_{0}^{T}\|\mathbf{y}(t)-\mathbf{V} \widehat{\mathbf{y}}(t)\|^{2} d t \leq \mathbf{C}\left(\mathcal{E}_{\mathbf{y}}+\mathcal{E}_{\mathbf{f}}\right)
$$

where $\mathbf{C}:=\max \left\{1+c_{\mu} \alpha^{2} T, c_{\mu} \beta^{2} T\right\}$,

$$
\begin{aligned}
& \alpha:=\left\|\mathbf{V}^{T} \mathbf{A}\right\|+\left\|\mathbf{V}^{T} \mathbb{P}\right\| L_{f}, \quad \beta:=\left\|\mathbf{V}^{T}(\mathbf{I}-\mathbb{P})\right\|, \quad \gamma:=\left\|\mathbf{V}^{T} \mathbb{P}\right\| L_{f}, \\
& c_{\mu}:=\widetilde{Q}_{\mu} e^{2 \gamma Q_{\mu}}, \text { with } \begin{cases}Q_{\mu}=\frac{1}{\mu}\left(e^{\mu T}-1\right), \widetilde{Q}_{\mu}=\frac{1}{\mu}\left(e^{2 \mu T}-1\right), & \mu \neq 0 \\
Q_{\mu}=\widetilde{Q}_{\mu}=2 T & , \quad \mu=0\end{cases} \\
& \mathcal{E}_{\mathbf{y}}:=\int_{0}^{T}\left\|\mathbf{y}(t)-\mathbf{V} \mathbf{V}^{T} \mathbf{y}(t)\right\|^{2} d t, \quad \mathcal{E}_{\mathbf{f}}:=\int_{0}^{T}\left\|\mathbf{f}(t)-\mathbf{U U}^{T} \mathbf{f}(t)\right\|^{2} d t
\end{aligned}
$$

with $\mathbf{f}(t)=\mathbf{F}(t, \mathbf{y}(t))$. In addition,

(i) if $\mu<0$, then $Q_{\mu}, \widetilde{Q}_{\mu}<\frac{1}{|\mu|}$ and $c_{\mu}$ in (3.9) can be bounded by a constant independent of $T$, i.e.

$$
c_{\mu}<\frac{1}{|\mu|} e^{2 \gamma /|\mu|}
$$

(ii) if the POD-DEIM reduced system (2.3) is constructed from the POD basis matrices $\mathbf{V} \in \mathbb{R}^{n \times k}$, and $\mathbf{U} \in \mathbb{R}^{n \times m}$ of solution snapshots and nonlinear snapshots, respectively, which satisfy (2.6), then, from (2.7) and (2.9),

$$
\mathcal{E}_{\mathbf{y}}=\sum_{\ell=k+1}^{r} \lambda_{\ell}^{\infty}, \quad \mathcal{E}_{\mathbf{f}}=\sum_{\ell=m+1}^{r_{s}} s_{\ell}^{\infty}
$$


Proof. Define the pointwise error $\mathbf{e}(t):=\mathbf{y}(t)-\mathbf{V} \widehat{\mathbf{y}}(t)$, and write

$$
\mathbf{e}(t)=\rho(t)+\theta(t)
$$

where $\rho(t):=\mathbf{y}(t)-\mathbf{V} \mathbf{V}^{T} \mathbf{y}(t), \quad \theta(t):=\mathbf{V} \mathbf{V}^{T} \mathbf{y}(t)-\mathbf{V} \widehat{\mathbf{y}}(t)$. The proof consists of developing a bound for $\|\theta(t)\|$ using Gronwall's lemma. Consider $\dot{\theta}(t)=\mathbf{V V}^{T} \dot{\mathbf{y}}(t)-$ V $\dot{\hat{\mathbf{y}}}(t)$ with $\dot{\mathbf{y}}(t)$ and $\dot{\hat{\mathbf{y}}}(t)$ satisfying (2.1) and (2.3). Then

$$
\dot{\theta}(t)=\mathbf{V} \mathbf{V}^{T}[\mathbf{A}[\rho(t)+\theta(t)]+\mathbf{F}(t, \mathbf{y}(t))-\mathbb{P} \mathbf{F}(t, \mathbf{V} \widehat{\mathbf{y}}(t))] .
$$

Define $\widehat{\theta}(t):=\mathbf{V}^{T} \theta(t)$. Pre-multiplying by $\mathbf{V}^{T}$ throughout (3.13), using orthonormality of the columns of $\mathbf{V}$, and noting that $\theta(t)=\mathbf{V} \widehat{\theta}(t)$ provides

$$
\frac{d}{d t} \widehat{\theta}(t)=\widehat{\mathbf{A}} \widehat{\theta}(t)+\mathbf{G}(t),
$$

where $\mathbf{G}(t):=\mathbf{V}^{T} \mathbf{A} \rho(t)+\mathbf{V}^{T}[\mathbf{F}(t, \mathbf{y}(t))-\mathbb{P} \mathbf{F}(t, \mathbf{V} \widehat{\mathbf{y}}(t))]$. Note that $\theta(0)=0$ since $\widehat{\mathbf{y}}_{0}=\mathbf{V}^{T} \mathbf{y}(0)$. Hence, the solution to (3.14) can be written as

$$
\widehat{\theta}(t)=\int_{0}^{t} e^{\widehat{\mathbf{A}}(t-s)} \mathbf{G}(s) d s .
$$

The Lipschitz continuity of $\mathbf{F}$ together with $(\mathbf{I}-\mathbb{P}) \mathbf{F}(t, \mathbf{y}(t))=(\mathbf{I}-\mathbb{P}) \mathbf{w}(t)$ from $(2.11)$ in Lemma 2.1, where $\mathbf{w}(t):=\mathbf{F}(t, \mathbf{y}(t))-\mathbf{U U}^{T} \mathbf{F}(t, \mathbf{y}(t))$, implies

$$
\begin{aligned}
& \|\mathbf{G}(t)\| \leq\left\|\mathbf{V}^{T} \mathbf{A} \rho(t)\right\|+\left\|\mathbf{V}^{T}(\mathbf{I}-\mathbb{P}) \mathbf{F}(t, \mathbf{y}(t))\right\|+\left\|\mathbf{V}^{T} \mathbb{P}\right\|\|\mathbf{F}(t, \mathbf{y}(t))-\mathbf{F}(t, \mathbf{V} \widehat{\mathbf{y}}(t))\| \\
& \leq \alpha\|\rho(t)\|+\beta\|\mathbf{w}(t)\|+\gamma\|\theta(t)\|, \\
& \text { where } \quad \alpha:=\left\|\mathbf{V}^{T} \mathbf{A}\right\|+\left\|\mathbf{V}^{T} \mathbb{P}\right\| L_{f}, \quad \beta:=\left\|\mathbf{V}^{T}(\mathbf{I}-\mathbb{P})\right\|, \quad \gamma:=\left\|\mathbf{V}^{T} \mathbb{P}\right\| L_{f} \text {. Since } \\
& \|\widehat{\theta}(t)\|=\|\theta(t)\| \text { and }\left\|e^{\widehat{\mathbf{A}}(t-s)}\right\| \leq e^{\mu(t-s)},(3.15) \text { and (3.16) imply } \\
& \|\theta(t)\| \leq \int_{0}^{t}\left\|e^{\widehat{\mathbf{A}}(t-s)}\right\|(\alpha\|\rho(s)\|+\beta\|\mathbf{w}(s)\|+\gamma\|\theta(s)\|) d s \\
& \leq \eta+\gamma \int_{0}^{t} e^{\mu(t-s)}\|\theta(s)\| d s
\end{aligned}
$$

where $\eta$ satisfies $\eta \geq \widehat{\eta}(t):=\int_{0}^{t} e^{\mu(t-s)}(\alpha\|\rho(s)\|+\beta\|\mathbf{w}(s)\|) d s$, for all $t \in[0, T]$. Applying the integral form of Gronwall's inequality [2] to (3.17) gives

$$
\|\theta(t)\| \leq \eta e^{\gamma q_{\mu}(t)}
$$

where $q_{\mu}(t):=\int_{0}^{t} e^{\mu(t-s)} d s$. Now, $\eta$ can be specified by applying the Cauchy-Schwarz inequality to $\widehat{\eta}(t)$ so that we can put

$$
\eta:=\left[\tilde{q}_{\mu}(T)\left(\alpha^{2} \int_{0}^{T}\|\rho(t)\|^{2} d t+\beta^{2} \int_{0}^{T}\|\mathbf{w}(t)\|^{2} d t\right)\right]^{1 / 2} .
$$

where $\tilde{q}_{\mu}(t):=2 \int_{0}^{t} e^{2 \mu(t-s)} d s=\left\{\begin{array}{ll}\frac{1}{\mu}\left(e^{2 \mu t}-1\right) & , \quad \mu \neq 0 \\ 2 t & , \quad \mu=0\end{array}\right.$. 
Using $q_{\mu}(t) \leq q_{\mu}(T)$ for all $t \in[0, T]$ and (3.18), a bound for $\|\theta(t)\|^{2}$ is given by

$$
\|\theta(t)\|^{2} \leq \tilde{q}_{\mu}(T) e^{2 \gamma q_{\mu}(T)}\left(\alpha^{2} \int_{0}^{T}\|\rho(t)\|^{2} d t+\beta^{2} \int_{0}^{T}\|\mathbf{w}(t)\|^{2} d t\right),
$$

for all $t \in[0, T]$. Finally, the proof is completed by applying the above bound to $\int_{0}^{T}\|\theta(t)\|^{2}$ on the right in $\int_{0}^{T}\|e(t)\|^{2} d t=\int_{0}^{T}\|\rho(t)\|^{2} d t+\int_{0}^{T}\|\theta(t)\|^{2} d t$. $\mathbf{\square}$

REMARK 3.2. The bound for the pointwise error $\mathbf{e}(t)=\|\mathbf{y}(t)-\mathbf{V} \widehat{\mathbf{y}}(t)\|$ for $t \in$ $[0, T]$ can be obtained directly from the proof of Theorem 3.1. For the special case when (i) and (ii) of Theorem 3.1 hold true, i.e. when $\mu<0$ and $\mathcal{E}_{\mathbf{y}}=\sum_{\ell=k+1}^{r} \lambda_{\ell}^{\infty}, \mathcal{E}_{\mathbf{f}}=$ $\sum_{\ell=m+1}^{r_{s}} s_{\ell}^{\infty}$, the pointwise error is uniformly bounded on $[0, T]$ :

$$
\|\mathbf{y}(t)-\mathbf{V} \widehat{\mathbf{y}}(t)\|^{2} \leq c\left(\sum_{\ell=k+1}^{r} \lambda_{\ell}^{\infty}+\sum_{\ell=m+1}^{r_{s}} s_{\ell}^{\infty}\right), \quad \text { for all } t \in[0, T],
$$

where $c:=2 \max \left\{1+c_{\mu} \alpha^{2}, c_{\mu} \beta^{2}\right\}, c_{\mu}=\frac{1}{|\mu|} e^{2 \gamma /|\mu|}$, with $\alpha, \beta$ defined as in (3.8).

From Theorem 3.1, $\mathcal{E}_{\mathbf{y}}$ and $\mathcal{E}_{\mathbf{f}}$ in (3.10) are the errors from approximating the solution $\mathbf{y}(t)$ and the nonlinear function $\mathbf{F}(t, \mathbf{y}(t))$ by orthogonal projections onto column spaces of $\mathbf{V}$ and $\mathbf{U}$, respectively. These errors are minimized in the case of (ii) in Theorem 3.1 and therefore (3.12) gives lower bounds for $\mathcal{E}_{\mathbf{y}}$ and $\mathcal{E}_{\mathbf{f}}$. The magnification factor $\mathbf{C}$ depends on the original problem through the quantities $\mathbf{A}, \mu(\mathbf{A}), L_{f}$ as well as the POD and DEIM approximations through $\mathbf{V}$ and $\mathbb{P}$.

The error analysis in this section has illustrated the basic idea concerning how the parabolicity assumption together with the combination of the POD-DEIM approach will lead to a bound on the state approximation error. However, it depends upon the ability to separate out a constant matrix $\mathbf{A}$ on the right hand side of the ODE system. The key tool in this analysis has been the logarithmic norm. In the next section, we shall utilize a generalization to obtain an error estimate that does not require the constant matrix A.

4. Analysis based on generalized logarithmic norm. A logarithmic norm was used in the previous section to analyze the state approximation error of the PODDEIM system. That approach required the presence of a constant matrix A. More generally, as is done in [27], one can apply a logarithmic norm argument to a local linearization about the trajectory. In the remainder of our analysis, we shall employ a generalization of the logarithmic norm that avoids the need for a linearization or for the presence of a constant $\mathbf{A}$. The generalization of logarithmic norm to unbounded nonlinear operators was introduced through logarithmic Lipschitz constants in [26] to avoid working with linearizations and logarithmic norms that are only applicable to bounded operators. Here, we shall use this analysis tool to develop a conceptual framework suitable to analyzing POD-DEIM reduced systems of nonlinear ODEs. We will now consider nonlinear ODEs of the form:

$$
\dot{\mathbf{y}}(t)=\mathbf{F}(t, \mathbf{y}(t)), \quad \mathbf{y}(0)=\mathbf{y}_{0},
$$

where $\mathbf{F}:[0, T] \times \mathcal{Y} \rightarrow \mathbb{R}^{n}, \mathcal{Y} \subseteq \mathbb{R}^{n}$ with POD-DEIM reduced system of the form:

$$
\dot{\hat{\mathbf{y}}}(t)=\widehat{\mathbf{F}}(t, \widehat{\mathbf{y}}(t)), \quad \widehat{\mathbf{y}}(0)=\mathbf{V}^{T} \mathbf{y}_{0},
$$

where $\widehat{\mathbf{F}}:[0, T] \times \widehat{\mathcal{Y}} \rightarrow \mathbb{R}^{k}, \widehat{\mathcal{Y}} \subseteq \mathbb{R}^{k}, \widehat{\mathbf{F}}(t, \widehat{\mathbf{y}})=\mathbf{V}^{T} \mathbb{P} \mathbf{F}(t, \mathbf{V} \widehat{\mathbf{y}}(t))$ for $\widehat{\mathbf{y}}(t) \in \widehat{\mathcal{Y}}, t \in[0, T]$. Note that the POD reduced system can be obtained by replacing $\mathbb{P}$ with the $n$-by- $n$ 
identity matrix. Hence, the error bounds derived in this section also apply to the POD reduced system. We shall work with the Euclidian inner product $\langle\cdot, \cdot\rangle: \mathbb{R}^{d} \times \mathbb{R}^{d} \rightarrow \mathbb{R}$, for some positive integer $d$, i.e. $\langle\mathbf{u}, \mathbf{v}\rangle=\mathbf{u}^{T} \mathbf{v}$ for $\mathbf{u}, \mathbf{v} \in \mathbb{R}^{d}$, and its induced norm $\|\mathbf{u}\|=\sqrt{\langle\mathbf{u}, \mathbf{u}\rangle}, \mathbf{u} \in \mathbb{R}^{d}$. As in [27], for a map $\mathbf{F}:[0, T] \times \mathcal{Y} \rightarrow \mathbb{R}^{d}, \mathcal{Y} \subseteq \mathbb{R}^{d}$, the least upper bound (lub) logarithmic Lipschitz constants with respect to the inner product $\langle\cdot, \cdot\rangle$ can be defined, uniformly for all $t \in[0, T]$, as:

$$
M[\mathbf{F}]:=\sup _{\mathbf{u} \neq \mathbf{v}} \frac{\langle\mathbf{u}-\mathbf{v}, \mathbf{F}(t, \mathbf{u})-\mathbf{F}(t, \mathbf{v})\rangle}{\|\mathbf{u}-\mathbf{v}\|^{2}} .
$$

The convergence of the solution as well as the stability of the corresponding PODDEIM reduced system can be analyzed by using these logarithmic Lipschitz constants. The map $\mathbf{F}$ is called uniformly negative monotone if $M[\mathbf{F}]<0$, in which case it will be shown that the error bound of the reduced-order solution is uniformly bounded on $t \in[0, T]$.

The asymptotic error analysis will be considered first in $\S 4.1$ for the continuous setting where the overall accuracy of the reduced system is only contributed from applying the POD-DEIM technique without other effects such as the choice of time integration method. Then, a framework for error analysis in the discrete setting for the implicit Euler time integration scheme will be presented in $\S 4.2$. The proposed error bounds in both continuous and discrete settings are derived through an application of generalized logarithmic norms discussed earlier and they are summarized in Theorem 4.1. Note that Lipschitz continuity of $\mathbf{F}$ is the only main assumption used in Theorem 4.1. The resulting error bounds in 2-norm reflect the approximation property of POD based scheme through the decay of singular values.

THEOREM 4.1. Let $\mathbf{y}(t)$ be the solution of the original full-order system (4.1) and $\widehat{\mathbf{y}}$ be the solution of the POD-DEIM reduced system (4.2), for $t \in[0, T]$. Let $Y_{j}$ and $\widehat{Y}_{j}$ be the solutions of the discretized systems of (4.1) and (4.2), respectively, obtained from implicit Euler time integration at $t_{j}=\Delta t j \in[0, T], \triangle t=T / n_{t}$ for $j=0, \ldots n_{t}$. Let $M[\widehat{\mathbf{F}}]$ be the logarithmic Lipschitz constant of $\widehat{\mathbf{F}}$ defined as in (4.3) and assume that $\mathbf{F}(t, \mathbf{y})$ in (4.1) is Lipschitz continuous with Lipschitz constant $L_{f}$ as in (2.2). Then

$$
\begin{array}{r}
\int_{0}^{T}\|\mathbf{y}(t)-\mathbf{V} \widehat{\mathbf{y}}(t)\|^{2} d t \leq \mathcal{C}\left(\mathcal{E}_{\mathbf{y}}+\mathcal{E}_{\mathbf{f}}\right), \\
\sum_{j=0}^{n_{t}}\left\|Y_{j}-\mathbf{V} \widehat{Y}_{j}\right\|^{2} \leq \overline{\mathcal{C}}\left(\overline{\mathcal{E}}_{\mathbf{y}}+\overline{\mathcal{E}}_{\mathbf{f}}\right),
\end{array}
$$

where $\mathcal{C}:=\max \left\{1+c_{M} \alpha^{2} T, c_{M} \beta^{2} T\right\}$ and $\overline{\mathcal{C}}:=\max \left\{1+\bar{c}_{M} \alpha^{2} T, \bar{c}_{M} \beta^{2} T\right\}$,

$$
\begin{aligned}
& \alpha:=\left\|\mathbf{V}^{T} \mathbb{P}\right\| L_{f}, \quad \beta:=\left\|\mathbf{V}^{T}(\mathbf{I}-\mathbb{P})\right\|, \quad \zeta:=\frac{1}{1-\triangle t M[\widehat{\mathbf{F}}]}, \\
& c_{M}:=\left\{\begin{array}{ll}
\frac{1}{M[\widehat{\mathbf{F}}]}\left(e^{2 M[\widehat{\mathbf{F}}] T}-1\right) & , \quad M[\widehat{\mathbf{F}}] \neq 0 \\
2 T & , \quad M[\widehat{\mathbf{F}}]=0
\end{array}, \bar{c}_{M}:=\Delta t \zeta^{2}\left(\frac{1-\zeta^{2 n_{t}}}{1-\zeta^{2}}\right),\right. \\
& \mathcal{E}_{\mathbf{y}}:=\int_{0}^{T}\left\|\mathbf{y}(t)-\mathbf{V} \mathbf{V}^{T} \mathbf{y}(t)\right\|^{2} d t, \quad \mathcal{E}_{\mathbf{f}}:=\int_{0}^{T}\left\|\mathbf{f}(t)-\mathbf{U} \mathbf{U}^{T} \mathbf{f}(t)\right\|^{2} d t, \\
& \overline{\mathcal{E}}_{\mathbf{y}}:=\sum_{j=0}^{n_{t}}\left\|Y_{j}-\mathbf{V} \mathbf{V}^{T} Y_{j}\right\|^{2}, \quad \overline{\mathcal{E}}_{\mathbf{f}}:=\sum_{j=0}^{n_{t}}\left\|F_{j}-\mathbf{U U}^{T} F_{j}\right\|^{2},
\end{aligned}
$$


with $\mathbf{f}(t)=\mathbf{F}(t, \mathbf{y}(t)), F_{j}=\mathbf{F}\left(t_{j}, Y_{j}\right)$. In addition,

(i) if $M[\widehat{\mathbf{F}}]<0$, then $c_{M}$ and $\bar{c}_{M}$ in (4.7) are bounded by

$$
c_{M}<\frac{1}{|M[\widehat{\mathbf{F}}]|} \text {, and } \quad \bar{c}_{M}<\frac{1}{|M[\widehat{\mathbf{F}}]|+\triangle t M[\widehat{\mathbf{F}}]^{2} / 2} ;
$$

(ii) if the POD basis matrices $\mathbf{V} \in \mathbb{R}^{n \times k}$ and $\mathbf{U} \in \mathbb{R}^{n \times m}$ used in (4.2), respectively, satisfy (2.7) and (2.9), then

$$
\mathcal{E}_{\mathbf{y}}=\sum_{\ell=k+1}^{r} \lambda_{\ell}^{\infty}, \quad \mathcal{E}_{\mathbf{f}}=\sum_{\ell=m+1}^{r_{s}} s_{\ell}^{\infty}
$$

(iii) if $\mathbf{V} \in \mathbb{R}^{n \times k}$ and $\mathbf{U} \in \mathbb{R}^{n \times m}$ used in (4.2) are the POD basis matrices of snapshot matrices $\mathbb{Y}=\left[Y_{1}, \ldots, Y_{n_{t}}\right]$ and $\mathbb{F}=\left[\mathbf{F}\left(t_{1}, Y_{1}\right), \ldots, \mathbf{F}\left(t_{n_{t}}, Y_{n_{t}}\right)\right] \in$ $\mathbb{R}^{n \times n_{t}}$, then using (2.5) and (2.8) gives

$$
\overline{\mathcal{E}}_{\mathbf{y}}=\sum_{\ell=k+1}^{\bar{r}} \lambda_{\ell}, \quad \overline{\mathcal{E}}_{\mathbf{f}}=\sum_{\ell=m+1}^{\bar{r}_{s}} s_{\ell} .
$$

The bounds for pointwise errors can be obtained similarly and are given below in some special cases.

REMARK 4.2. Using the notations and assumptions from Theorem 4.1:

- When the conditions (i) and (ii) of Theorem 4.1 hold true, the the norm of the pointwise error is uniformly bounded on $[0, T]$ :

$$
\|\mathbf{y}(t)-\mathbf{V} \widehat{\mathbf{y}}(t)\|^{2} \leq c\left(\sum_{\ell=k+1}^{r} \lambda_{\ell}^{\infty}+\sum_{\ell=m+1}^{r_{s}} s_{\ell}^{\infty}\right), \quad \text { for all } t \in[0, T]
$$

where $c=\max \left\{1+\alpha^{2} /|M[\widehat{\mathbf{F}}]|, \beta^{2} /|M[\widehat{\mathbf{F}}]|\right\}$.

- When (i) and (iii) of Theorem 4.1 hold true, the norm of the pointwise error in discrete setting is uniformly bounded at each time step:

$$
\left\|Y_{\ell}-\mathbf{V} \widehat{Y}_{\ell}\right\|^{2} \leq \bar{c}\left(\sum_{\ell=k+1}^{\bar{r}} \lambda_{\ell}+\sum_{\ell=m+1}^{\bar{r}_{s}} s_{\ell}\right), \quad \text { for all } \ell=1, \ldots n_{t}
$$

where $\bar{c}=\max \left\{1+\bar{q} \alpha^{2}, \bar{q} \beta^{2}\right\}, \bar{q}=\frac{1}{|M[\overline{\mathbf{F}}]|+\triangle t M[\overline{\mathbf{F}}]^{2} / 2}$.

Notice that , for $M[\mathbf{F}]<0$, the error bound (4.5) in the discretized setting converges to the bound (4.4) in the continuous setting. In particular, as $\Delta t \rightarrow 0$, it was shown in [17] that $\overline{\mathcal{E}}_{\mathbf{y}}$ and $\overline{\mathcal{E}}_{\mathbf{f}}$ converge to $\mathcal{E}_{\mathbf{y}}$ and $\mathcal{E}_{\mathbf{f}}$, respectively; and from (4.10), we have that the bound for $\bar{c}_{M}$ converges to the bound for $c_{M}$.

Notice also that there are two main differences for the error bounds in the continuous setting from (3.7) of Theorem 3.1 and from (4.4) of Theorem 4.1: one in the quantities $\mu(\cdot)$ and $M[\cdot]$; and the other in the terms $c_{\mu}$ and $c_{M}$. Note that, $\mu(\cdot)$ and $M[\cdot]$ are the same when they are applied to linear operators, and hence there is no need to introduce the notion of logarithmic Lipschitz constant for linear systems. With nonlinearities, however, applying the logarithmic Lipschitz constant $M[\cdot]$ will 
allow us to avoid using Gronwall's inequality as required in the classical approach for deriving error bounds which often gives pessimistic bounds with exponential growth, e.g. the term $c_{\mu}$ in (3.11) has the exponential part, $e^{2 \gamma Q_{\mu}}$, arising from applying Gronwall's inequality in (3.18), while $c_{M}$ in (4.10) does not. The applications of $M[\cdot]$ for deriving the error bounds in Theorem 4.1 are presented next.

4.1. Error bounds in ODE setting. Consider the error of the solution from the POD-DEIM reduced system of the form

$$
\mathbf{e}(t)=\mathbf{y}(t)-\mathbf{y}_{r}(t), \quad \mathbf{y}_{r}(t):=\mathbf{V} \widehat{\mathbf{y}}(t)
$$

where $\mathbf{V} \in \mathbb{R}^{n \times k}$ be the POD basis matrix with $\mathbf{y}$ and $\widehat{\mathbf{y}}$ satisfying $\dot{\mathbf{y}}(t)=\mathbf{F}(t, \mathbf{y}(t)), \mathbf{y}(0)=$ $\mathbf{y}_{0}$, and $\dot{\hat{\mathbf{y}}}(t)=\mathbf{V}^{T} \mathbb{P} \mathbf{F}(t, \mathbf{V} \widehat{\mathbf{y}}(t)), \widehat{\mathbf{y}}(0)=\mathbf{V}^{T} \mathbf{y}_{0}$, for $t \in[0, T]$. Again, put

$$
\mathbf{e}(t)=\rho(t)+\theta(t)
$$

where $\rho(t):=\mathbf{y}(t)-\mathbf{V} \mathbf{V}^{T} \mathbf{y}(t), \theta(t):=\mathbf{V} \mathbf{V}^{T} \mathbf{y}(t)-\mathbf{V} \widehat{\mathbf{y}}(t)$, and note that $\widehat{\mathbf{y}}(0)=\mathbf{V}^{T} \mathbf{y}_{0}$ implies $\theta(0)=0$. Note also that since $\rho(t)^{T} \theta(t)=0$, $\|e(t)\|^{2}=\|\rho(t)\|^{2}+\|\theta(t)\|^{2}$. Define $\widehat{\theta}(t):=\mathbf{V}^{T} \theta(t)$. As before, $\theta(t)=\mathbf{V} \widehat{\theta}(t)$ and hence $\|\theta(t)\|=\|\widehat{\theta}(t)\|$. Now, consider

$$
\begin{aligned}
\mathbf{V}^{T} \dot{\mathbf{y}}(t) & =\widehat{\mathbf{F}}\left(t, \mathbf{V}^{T} \mathbf{y}(t)\right)+\widehat{\mathbf{r}}(t), \\
\dot{\hat{\mathbf{y}}}(t) & =\widehat{\mathbf{F}}(t, \widehat{\mathbf{y}}(t))
\end{aligned}
$$

where

$$
\widehat{\mathbf{r}}(t):=\mathbf{V}^{T} \mathbf{F}(t, \mathbf{y}(t))-\widehat{\mathbf{F}}\left(t, \mathbf{V}^{T} \mathbf{y}(t)\right) .
$$

This gives $\dot{\hat{\theta}}(t)=\mathbf{V}^{T} \dot{\mathbf{y}}(t)-\dot{\hat{\mathbf{y}}}(t)=\widehat{\mathbf{F}}\left(t, \mathbf{V}^{T} \mathbf{y}(t)\right)-\widehat{\mathbf{F}}(t, \widehat{\mathbf{y}}(t))+\widehat{\mathbf{r}}(t)$. Next, since $\|\widehat{\theta}(t)\|^{2}=\widehat{\theta}(t)^{T} \widehat{\theta}(t)$

$$
\begin{aligned}
\frac{d}{d t}\|\widehat{\theta}(t)\| & =\frac{\langle\widehat{\theta}(t), \dot{\hat{\theta}}(t)\rangle}{\|\widehat{\theta}(t)\|} \\
& =\frac{\left\langle\widehat{\theta}(t), \widehat{\mathbf{F}}\left(t, \mathbf{V}^{T} \mathbf{y}(t)\right)-\widehat{\mathbf{F}}(t, \widehat{\mathbf{y}}(t))+\widehat{\mathbf{r}}(t)\right\rangle}{\|\widehat{\theta}(t)\|} \\
& =\frac{\left\langle\widehat{\theta}(t), \widehat{\mathbf{F}}\left(t, \mathbf{V}^{T} \mathbf{y}(t)\right)-\widehat{\mathbf{F}}(t, \widehat{\mathbf{y}}(t))\right\rangle}{\|\widehat{\theta}(t)\|}+\frac{\langle\widehat{\theta}(t), \widehat{\mathbf{r}}(t)\rangle}{\|\widehat{\theta}(t)\|} \\
& \leq M[\widehat{\mathbf{F}}]\|\widehat{\theta}(t)\|+\|\widehat{\mathbf{r}}(t)\| .
\end{aligned}
$$

Notice that $\|\widehat{\mathbf{r}}(t)\|$ is independent of $\|\widehat{\theta}(t)\|$ and hence Gronwall's inequality is not required here. Since $\|\theta(t)\|=\|\widehat{\theta}(t)\|$ and $\|\widehat{\theta}(t)\|=0$, we have

$$
\|\theta(t)\| \leq e^{M[\widehat{\mathbf{F}}] t}\|\theta(0)\|+\int_{0}^{t} e^{M[\widehat{\mathbf{F}}](t-\tau)}\|\widehat{\mathbf{r}}(\tau)\| d \tau=\int_{0}^{t} e^{M[\widehat{\mathbf{F}}](t-\tau)}\|\widehat{\mathbf{r}}(\tau)\| d \tau .
$$

We now recast the expression for $\widehat{\mathbf{r}}(t)$ as the sum of differences that can be estimated in terms of the neglected singular values. From (2.11) in Lemma 2.1, for $\mathbf{w}(t)=$ $\mathbf{F}(t, \mathbf{y}(t))-\mathbf{U U}^{T} \mathbf{F}(t, \mathbf{y}(t))$,

$$
\begin{aligned}
\widehat{\mathbf{r}}(t) & =\mathbf{V}^{T} \mathbf{F}(t, \mathbf{y}(t))-\widehat{\mathbf{F}}\left(t, \mathbf{V}^{T} \mathbf{y}(t)\right)=\mathbf{V}^{T}\left[\mathbf{F}(t, \mathbf{y}(t))-\mathbb{P} \mathbf{F}\left(t, \mathbf{V} \mathbf{V}^{T} \mathbf{y}(t)\right)\right] \\
& =\mathbf{V}^{T}\left[\mathbf{F}(t, \mathbf{y}(t))-\mathbb{P} \mathbf{F}(t, \mathbf{y}(t))+\mathbb{P} \mathbf{F}(t, \mathbf{y}(t))-\mathbb{P} \mathbf{F}\left(t, \mathbf{V} \mathbf{V}^{T} \mathbf{y}(t)\right)\right] \\
& =\mathbf{V}^{T}(\mathbf{I}-\mathbb{P}) \mathbf{w}(t)+\mathbf{V}^{T} \mathbb{P}\left(\mathbf{F}(t, \mathbf{y}(t))-\mathbf{F}\left(t, \mathbf{V} \mathbf{V}^{T} \mathbf{y}(t)\right)\right) .
\end{aligned}
$$


The Lipschitz continuity of $\mathbf{F}$ implies $\left\|\mathbf{F}(t, \mathbf{y}(t))-\mathbf{F}\left(t, \mathbf{V V}^{T} \mathbf{y}(t)\right)\right\| \leq L_{f} \| \mathbf{y}(t)-$ $\mathbf{V V}^{T} \mathbf{y}(t)\left\|=L_{f}\right\| \rho(t) \|$, so that

$$
\|\widehat{\mathbf{r}}(t)\| \leq \alpha\|\rho(t)\|+\beta\|\mathbf{w}(t)\|,
$$

where $\alpha:=\left\|\mathbf{V}^{T} \mathbb{P}\right\| L_{f}, \beta:=\left\|\mathbf{V}^{T}(\mathbf{I}-\mathbb{P})\right\|$. Thus, for all $t \in[0, T]$ and for $\tilde{q}_{M}(t):=$ $\int_{0}^{t} e^{2 M[\widehat{\mathbf{F}}](t-\tau)} d \tau=\left\{\begin{array}{ll}\frac{1}{2 M[\widehat{\mathbf{F}}]}\left(e^{2 M[\widehat{\mathbf{F}}] t}-1\right), & M[\widehat{\mathbf{F}}] \neq 0 \\ t, & M[\widehat{\mathbf{F}}]=0\end{array}\right.$,

$$
\|\theta(t)\|^{2} \leq \tilde{q}_{M}(t) \int_{0}^{t}\|\widehat{\mathbf{r}}(\tau)\|^{2} d \tau \leq 2 \tilde{q}_{M}(T)\left(\alpha^{2} \int_{0}^{T}\|\rho(t)\|^{2} d t+\beta^{2} \int_{0}^{T}\|\mathbf{w}(t)\|^{2} d t\right) .
$$

Using the above bound for $\|\theta(t)\|$ with $\int_{0}^{T}\|e(t)\|^{2} d t=\int_{0}^{T}\|\rho(t)\|^{2} d t+\int_{0}^{T}\|\theta(t)\|^{2} d t$ will give (4.4) in Theorem 4.1. In the case when $\int_{0}^{T}\|\rho(t)\|^{2} d t=\sum_{\ell=k+1}^{r} \lambda_{\ell}^{\infty}$ and $\int_{0}^{T}\|\mathbf{w}(t)\|^{2} d t=\sum_{\ell=m+1}^{r_{s}} s_{\ell}^{\infty}$,

$$
\int_{0}^{T}\|e(t)\|^{2} d t=\int_{0}^{T}\|\rho(t)\|^{2} d t+\int_{0}^{T}\|\theta(t)\|^{2} d t \leq C\left(\sum_{\ell=k+1}^{r} \lambda_{\ell}^{\infty}+\sum_{\ell=m+1}^{r_{s}} s_{\ell}^{\infty}\right),
$$

where $C=\max \left\{1+2 T \tilde{q}_{M}(T) \alpha^{2}, 2 T \tilde{q}_{M}(T) \beta^{2}\right\}$. When $M[\widehat{\mathbf{F}}]<0, \tilde{q}_{M}(T) \leq \frac{1}{2|M[\widehat{\mathbf{F}}]|}$. Therefore the norm of the total pointwise error $\|e(t)\|$ is uniformly bounded on $[0, T]$ :

$$
\|e(t)\|^{2}=\left(\|\rho(t)\|^{2}+\|\theta(t)\|^{2}\right) \leq c\left(\sum_{\ell=k+1}^{r} \lambda_{\ell}^{\infty}+\sum_{\ell=m+1}^{r_{s}} s_{\ell}^{\infty}\right),
$$

where $c=\max \left\{1+\alpha^{2} /|M[\widehat{\mathbf{F}}]|, \beta^{2} /|M[\widehat{\mathbf{F}}]|\right\}$ as stated in (4.13) from Remark 4.2.

4.2. Error bounds in discretized ODE setting. Using our analysis of the full trajectory as a guide, by analogy to (4.1) and (4.2), we shall analyze the discrete systems obtained from backward Euler time integration corresponding to the fullorder system and the POD-DEIM reduced system in the form:

$$
\frac{Y_{j}-Y_{j-1}}{\triangle t}=\mathbf{F}\left(t_{j}, Y_{j}\right), \quad \frac{\widehat{Y}_{j}-\widehat{Y}_{j-1}}{\triangle t}=\widehat{\mathbf{F}}\left(t_{j}, \widehat{Y}_{j}\right),
$$

$\triangle t=T / n_{t}$, where $n_{t}$ is the number of time steps, $Y_{j}$ and $\widehat{Y}_{j}$ are approximations of $\mathbf{y}\left(t_{j}\right)$ and $\widehat{\mathbf{y}}\left(t_{j}\right)$ respectively, at $t_{j}=j \Delta t, j=0, \ldots, n_{t}$. Consider the error:

$$
E_{j}=Y_{j}-\mathbf{V} \widehat{Y}_{j}
$$

where $Y_{j}$ is the solution of full-order system $\widehat{Y}_{j}$ is the solution of the POD-DEIM reduced system in (4.20), for $j=1, \ldots, n_{t}$. Write

$$
E_{j}=\rho_{j}+\theta_{j},
$$

where $\rho_{j}:=Y_{j}-\mathbf{V} \mathbf{V}^{T} Y_{j}, \theta_{j}:=\mathbf{V} \mathbf{V}^{T} Y_{j}-\mathbf{V} \widehat{Y}_{j}$. Define $\widehat{\theta}_{j}:=\mathbf{V}^{T} \theta_{j}$. As before, $\theta_{j}=\mathbf{V} \widehat{\theta}_{j},\left\|\theta_{j}\right\|=\left\|\widehat{\theta}_{j}\right\|$ and $\rho_{j}^{T} \theta_{j}=0$. 
From (4.20), we have $\mathbf{V}^{T}\left(\frac{Y_{j}-Y_{j-1}}{\Delta t}\right)=\mathbf{V}^{T} \mathbf{F}\left(t_{j}, Y_{j}\right)$, and we shall consider

$$
\begin{aligned}
\mathbf{V}^{T}\left(\frac{Y_{j}-Y_{j-1}}{\triangle t}\right) & =\widehat{\mathbf{F}}\left(t_{j}, \mathbf{V}^{T} Y_{j}\right)+\widehat{R}_{j}, \\
\frac{\widehat{Y}_{j}-\widehat{Y}_{j-1}}{\triangle t} & =\widehat{\mathbf{F}}\left(t_{j}, \widehat{Y}_{j}\right),
\end{aligned}
$$

where

$$
\widehat{R}_{j}=\mathbf{V}^{T} \mathbf{F}\left(t_{j}, Y_{j}\right)-\widehat{\mathbf{F}}\left(t_{j}, \mathbf{V}^{T} Y_{j}\right) .
$$

So that for $\widehat{\theta}_{j}=\mathbf{V}^{T} Y_{j}-\widehat{Y}_{j}, \frac{\widehat{\theta}_{j}-\widehat{\theta}_{j-1}}{\Delta t}=\widehat{\mathbf{F}}\left(t_{j}, \mathbf{V}^{T} Y_{j}\right)-\widehat{\mathbf{F}}\left(t_{j}, \widehat{Y}_{j}\right)+\widehat{R}_{j}$. Then,

$$
\begin{aligned}
\frac{\left\|\widehat{\theta}_{j}\right\|-\left\|\widehat{\theta}_{j-1}\right\|}{\Delta t} & \leq \frac{1}{\Delta t}\left(\frac{\left\langle\widehat{\theta}_{j}, \widehat{\theta}_{j}\right\rangle}{\left\|\widehat{\theta}_{j}\right\|}-\frac{\left\langle\widehat{\theta}_{j}, \widehat{\theta}_{j-1}\right\rangle}{\left\|\widehat{\theta}_{j}\right\|}\right) \\
& =\frac{1}{\left\|\widehat{\theta}_{j}\right\|}\left\langle\widehat{\theta}_{j}, \frac{\widehat{\theta}_{j}-\widehat{\theta}_{j-1}}{\Delta t}\right\rangle \\
& =\frac{1}{\left\|\widehat{\theta}_{j}\right\|}\left\langle\widehat{\theta}_{j}, \widehat{\mathbf{F}}\left(t_{j}, \mathbf{V}^{T} Y_{j}\right)-\widehat{\mathbf{F}}\left(t_{j}, \widehat{Y}_{j}\right)+\widehat{R}_{j}\right\rangle \\
& =\frac{1}{\left\|\widehat{\theta}_{j}\right\|}\left\langle\theta_{j}, \widehat{\mathbf{F}}\left(t_{j}, \mathbf{V}^{T} Y_{j}\right)-\widehat{\mathbf{F}}\left(t_{j}, \widehat{Y}_{j}\right)\right\rangle+\frac{1}{\left\|\widehat{\theta}_{j}\right\|}\left\langle\widehat{\theta}_{j}, \widehat{R}_{j}\right\rangle \\
& \leq M[\widehat{\mathbf{F}}]\left\|\widehat{\theta}_{j}\right\|+\left\|\widehat{R}_{j}\right\|,
\end{aligned}
$$

where we have used $\left\langle\widehat{\theta}_{j}, \widehat{\theta}_{j-1}\right\rangle \leq\left\|\widehat{\theta}_{j}\right\|\left\|\widehat{\theta}_{j-1}\right\|$ in the first inequality and used $\left\langle\widehat{\theta}_{j}, \widehat{\mathbf{F}}\left(\mathbf{V}^{T} Y_{j}\right)-\right.$ $\left.\widehat{\mathbf{F}}\left(\widehat{Y}_{j}\right)\right\rangle \leq M[\widehat{\mathbf{F}}]\left\|\widehat{\theta}_{j}\right\|^{2}$ from (4.3) and $\left\langle\widehat{\theta}_{j}, \widehat{R}_{j}\right\rangle \leq\left\|\widehat{\theta}_{j}\right\|\left\|\widehat{R}_{j}\right\|$ in the last inequality. That is, using $\left\|\widehat{\theta}_{j}\right\|=\left\|\theta_{j}\right\|$ for $\zeta:=\frac{1}{1-\triangle t M[\hat{\mathbf{F}}]}$,

$\left\|\theta_{j}\right\| \leq \zeta\left(\left\|\theta_{j-1}\right\|+\Delta t\left\|\widehat{R}_{j}\right\|\right) \leq \zeta^{j}\left\|\theta_{0}\right\|+\Delta t \sum_{\ell=1}^{j} \zeta^{\ell}\left\|\widehat{R}_{j-\ell+1}\right\| \leq \Delta t\left(q_{j} \sum_{\ell=1}^{j}\left\|\widehat{R}_{\ell}\right\|^{2}\right)^{1 / 2}$

where $q_{j}:=\sum_{\ell=1}^{j} \zeta^{2 \ell}$ and we have used $\theta_{0}=0$. As in the continuous case, we recast $\left\|\widehat{R}_{\ell}\right\|$ as a sum of differences that can be estimated using the neglected singular values.

$$
\begin{aligned}
\widehat{R}_{\ell} & =\mathbf{V}^{T} \mathbf{F}\left(t_{\ell}, Y_{\ell}\right)-\widehat{\mathbf{F}}\left(t_{\ell}, \mathbf{V} \mathbf{V}^{T} Y_{\ell}\right)=\mathbf{V}^{T}\left[\mathbf{F}\left(t_{\ell}, Y_{\ell}\right)-\mathbb{P} \mathbf{F}\left(t_{\ell}, \mathbf{V} \mathbf{V}^{T} Y_{\ell}\right)\right] \\
& =\mathbf{V}^{T}\left[\mathbf{F}\left(t_{\ell}, Y_{\ell}\right)-\mathbb{P} \mathbf{F}\left(t_{\ell}, Y_{\ell}\right)+\mathbb{P} \mathbf{F}\left(t_{\ell}, Y_{\ell}\right)-\mathbb{P} \mathbf{F}\left(t_{\ell}, \mathbf{V V}^{T} Y_{\ell}\right)\right] \\
& =\mathbf{V}^{T}(\mathbf{I}-\mathbb{P}) \mathbf{w}_{\ell}+\mathbf{V}^{T} \mathbb{P}\left(\mathbf{F}\left(t_{\ell}, Y_{\ell}\right)-\mathbf{F}\left(t_{\ell}, \mathbf{V} \mathbf{V}^{T} Y_{\ell}\right)\right),
\end{aligned}
$$

where $\mathbf{w}_{\ell}=\left(\mathbf{I}-\mathbf{U U}^{T}\right) \mathbf{F}\left(t_{\ell}, Y_{\ell}\right)$ from (2.11) in Lemma 2.1. The Lipschitz continuity of $\mathbf{F}$ implies $\left\|\mathbf{F}\left(t_{\ell}, Y_{\ell}\right)-\mathbf{F}\left(t_{\ell}, \mathbf{V} \mathbf{V}^{T} Y_{\ell}\right)\right\| \leq L_{f}\left\|Y_{\ell}-\mathbf{V V}^{T} Y_{\ell}\right\|=L_{f}\left\|\rho_{\ell}\right\|$, and thus

$$
\left\|\widehat{R}_{\ell}\right\| \leq \alpha\left\|\rho_{\ell}\right\|+\beta\left\|\mathbf{w}_{\ell}\right\|,
$$

where $\alpha:=\left\|\mathbf{V}^{T} \mathbb{P}\right\| L_{f}, \beta:=\left\|\mathbf{V}^{T}(\mathbf{I}-\mathbb{P})\right\|$. Let $q:=q_{n_{t}}=\sum_{\ell=1}^{n_{t}} \zeta^{2 \ell}$, so for $j=$ $0, \ldots, n_{t}$,

$$
\left\|\theta_{j}\right\|^{2} \leq \Delta t^{2} q\left(\sum_{\ell=1}^{j}\left\|\widehat{R}_{\ell}\right\|^{2}\right) \leq \Delta t^{2} 2 q\left(\alpha^{2} \sum_{\ell=1}^{j}\left\|\rho_{\ell}\right\|^{2}+\beta^{2} \sum_{\ell=1}^{j}\left\|\mathbf{w}_{\ell}\right\|^{2}\right) .
$$


Applying the above bound for $\left\|\theta_{j}\right\|$ to $\sum_{\ell=0}^{n_{t}}\left\|E_{\ell}\right\|^{2}=\sum_{\ell=0}^{n_{t}}\left\|\rho_{\ell}\right\|^{2}+\sum_{\ell=0}^{n_{t}}\left\|\theta_{\ell}\right\|^{2}$ gives the error bound (4.5) in Theorem 4.1. In the case when $\sum_{\ell=0}^{n_{t}}\left\|\rho_{\ell}\right\|^{2}=\sum_{\ell=k+1}^{r} \lambda_{\ell}$ and $\sum_{\ell=0}^{n_{t}}\left\|\mathbf{w}_{\ell}\right\|^{2}=\sum_{\ell=m+1}^{r_{s}} s_{\ell}$,

$$
\sum_{\ell=0}^{n_{t}}\left\|E_{\ell}\right\|^{2}=\sum_{\ell=0}^{n_{t}}\left\|\rho_{\ell}\right\|^{2}+\sum_{\ell=0}^{n_{t}}\left\|\theta_{\ell}\right\|^{2} \leq \bar{C}\left(\sum_{\ell=k+1}^{r} \lambda_{\ell}+\sum_{\ell=m+1}^{r_{s}} s_{\ell}\right),
$$

where $\bar{C}=\max \left\{1+2 T q \triangle t \alpha^{2}, 2 T q \triangle t \beta^{2}\right\}$ and for $T=n_{t} \Delta t$. When $M[\widehat{\mathbf{F}}]<0$, for all $j=1,2, \ldots, n_{t}, q_{j}=\sum_{\ell=1}^{j} \zeta^{2 \ell} \leq \sum_{\ell=1}^{\infty} \zeta^{2 \ell}=\sum_{\ell=0}^{\infty} \zeta^{2 \ell}-1=\frac{1}{1-\zeta^{2}}-1=$ $\frac{1}{(1-\Delta t M[\hat{\mathbf{F}}])^{2}-1}$. Therefore the norm of the total error $\left\|E_{j}\right\|$ is uniformly bounded on $[0, T]$ as shown below:

$$
\left\|E_{\ell}\right\|^{2}=\left\|\rho_{\ell}\right\|^{2}+\left\|\theta_{\ell}\right\|^{2} \leq \bar{c}\left(\sum_{\ell=k+1}^{r} \lambda_{\ell}+\sum_{\ell=m+1}^{r_{s}} s_{\ell}\right),
$$

where $\bar{c}=\max \left\{1+\bar{q} \alpha^{2}, \bar{q} \beta^{2}\right\}, \bar{q}=\frac{1}{|M[\widehat{\mathbf{F}}]|+\Delta t M[\widehat{\mathbf{F}}]^{2} / 2}$ as given in (4.14) of Remark 4.2.

5. Conclusion. This paper provides the error bounds of the state approximations from the POD-DEIM reduced systems for the ODEs with Lipschitz continuous nonlinearities. The asymptotic error analysis were considered in the continuous setting where we assumed the availability of the solutions on the entire time interval and the overall accuracy of the reduced system was only contributed from applying the POD-DEIM technique. This paper also presented a framework for error analysis in the discrete setting for the implicit Euler time integration scheme, which can be extended to other numerical methods. The proposed error bounds in both continuous and discrete settings were derived through a classical approach using logarithmic norms as well as through an application of generalized logarithmic norms [26]. The conditions under which the reduction error is uniformly bounded were also discussed. The resulting error bounds in 2-norm reflect the approximation property of POD based scheme through the decay of the corresponding singular values.

6. Acknowledgments. Part of this work was done during the visit of the authors at Delft University of Technology during fall 2010. We are grateful for the hospitality and the stimulating scientific atmosphere provided during our visit.

\section{REFERENCES}

[1] M. Barrault, Y. Maday, N. C. Nguyen, and A. T. Patera, An 'Empirical Interpolation' Method: Application to Efficient Reduced-Basis Discretization Of Partial Differential Equations, Comptes Rendus Mathematique, 339 (2004), pp. 667-672.

[2] R. Bellman, The stability of solutions of linear differential equations, Duke Math. J., 10 (1943), pp. 643-647.

[3] S. Chaturantabut and D. C. Sorensen, Application of POD and DEIM to Dimension Reduction of Nonlinear Miscible Viscous Fingering in Porous Media, Tech. Report TR09-25, CAAM, Rice U., July 2009.

[4] — Nonlinear model reduction via discrete empirical interpolation, SIAM Journal on Scientific Computing, 32 (2010), pp. 2737-2764.

[5] G. DAHLQUist, Stability and error bounds in the numerical integration of ordinary differential equations, Transactions of the Royal Institute of Technology 130, Stockholm, Sweden, (1959).

[6] G. DAhlquist, 33 years of numerical instability, part i, BIT Numerical Mathematics, 25 (1985), pp. 188-204. 10.1007/BF01934997. 
[7] J. L. Eftang, M. A. Grepl, and A. T. Patera, A posteriori error bounds for the empirical interpolation method, Comptes Rendus Mathematique, 348 (2010), pp. 575 - 579.

[8] F.Ebert, A note on pod model reduction methods for daes, Preprint 06-364 (Matheon),Inst. f. Mathematik, TU Berlin, (2006).

[9] D. Galbally, K. Fidkowski, K. Willcox, and O. Ghattas, Non-linear model reduction for uncertainty quantification in large-scale inverse problems, International Journal for Numerical Methods in Engineering, 81 (2010), pp. 1581-1608.

[10] M. A. Grepl, Reduced-basis Approximations and A Posteriori Error Estimation for Parabolic Partial Differential Equations, PhD thesis, Massachusetts Institute of Technology, 2005.

[11] T. H. Gronwall, Note on the derivatives with respect to a parameter of the solutions of a system of differential equations, The Annals of Mathematics, 20 (1919), pp. 292-296.

[12] M. Hinze AND M. Kunkel, Discrete empirical interpolation in pod model order reduction of drift-diffusion equations in electrical networks, Hamburger Beitrge zur Angewandten Mathematik, (2010).

[13] C. Homescu, L. R. Petzold, And R. Serban, Error estimation for reduced-order models of dynamical systems, SIAM Journal on Numerical Analysis, 43 (2005), pp. 1693-1714.

[14] — Error estimation for reduced-order models of dynamical systems, SIAM Review, 49 (2007), pp. 277-299.

[15] A. R. Kellems, S. Chaturantabut, D. C. Sorensen, and S. J. Cox, Morphologically accurate reduced order modeling of spiking neurons, Journal of Computational Neuroscience,DOI:10.1007/s10827-010-0229-4, (2010).

[16] K. Kunisch And S. Volkwein, Galerkin Proper Orthogonal Decomposition Methods for Parabolic Problem, Numerische Mathematik, 90 (2001), pp. 117-148.

[17] _ Galerkin Proper Orthogonal Decomposition Methods for a General Equation in Fluid Dynamics, SIAM J. Numer. Anal., 40 (2002), pp. 492-515.

[18] C. F. VAN LOAN, The sensitivity of the matrix exponential, SIAM Journal on Numerical Analysis, 14 (1977), pp. 971-981.

[19] M. Meyer AND H. G. Matthies, Efficient model reduction in non-linear dynamics using the karhunen-love expansion and dual-weighted-residual methods, Computational Mechanics, 31 (2003), pp. 179-191. 10.1007/s00466-002-0404-1.

[20] N. C. Nguyen, Reduced-Basis Approximation and A Posteriori Error Bounds for Nonaffine and Nonlinear Partial Differential Equations: Application to Inverse Analysis, PhD thesis, Singapore-MIT Alliance, National University of Singapore, 2005.

$[21]$ — A posteriori error estimation and basis adaptivity for reduced-basis approximation of nonaffine-parametrized linear elliptic partial differential equations, J. Comput. Phys., 227 (2007), pp. 983-1006.

[22] A. T. Patera and G. J. Rozza, Reduced Basis Approximation and A Posteriori Error Estimation for Parametrized Partial Differential Equations, Version 1.0, Copyright MIT, 2006. To appear in (tentative rubric) MIT Pappalardo Graduate Monographs in Mechanical Engineering.

[23] C. Prud'homme, D. V. Rovas, K. Veroy, L. Machiels, Y. Maday, A. T. Patera, and G. TURINICI, Reliable real-time solution of parametrized partial differential equations: Reduced-basis output bound methods, Journal of Fluids Engineering, 124 (2002), pp. 70-80.

[24] M. Rathinam and L. R. Petzold, A new look at proper orthogonal decomposition, SIAM Journal on Numerical Analysis, 41 (2003), pp. 1893-1925.

[25] G. RozzA, Reduced-basis methods for elliptic equations in sub-domains with a posteriori error bounds and adaptivity, Appl. Numer. Math., 55 (2005), pp. 403-424.

[26] G. SÖDERLIND, Bounds on nonlinear operators in finite-dimensional banach spaces, Numerische Mathematik, 50 (1986), pp. 27-44. 10.1007/BF01389666.

[27] — The logarithmic norm. history and modern theory, BIT Numerical Mathematics, 46 (2006), pp. 631-652. 10.1007/s10543-006-0069-9.

[28] T. Ström, On logarithmic norms, SIAM Journal on Numerical Analysis, 12 (1975), pp. 741753.

[29] S. Utku, J. L. M. Clemente, and M. Salama, Errors in reduction methods, Computers \& Structures, 21 (1985), pp. 1153-1157.

[30] S. Volkwein, Model reduction using proper orthogonal decomposition. Lecture note, April 2008. http://www.uni-graz.at/imawww/volkwein/POD.pdf. 